\title{
Efeitos sedativos da administração intranasal ou intramuscular de midazolam associado ou não ao butorfanol em periquito autraliano (Melopsitacus undulatus)*
}

\section{Sedative effects of intranasal or intramuscular administration of midazolam with or without butorphanol in budgerigards (Melopsitacus undulatus)}

\author{
Everton Alberto Leonardi da Silva, ${ }^{* *}$ Gustavo Aléssio Trevisan, ${ }^{* *}$ Anderson Luiz de Carvalho, ${ }^{* *}$ Rafael Messias Luiz ${ }^{* *}$
}

\begin{abstract}
Resumo
Frequentemente o médico-veterinário é requisitado para realização de diversos procedimentos em aves, os quais geralmente necessitam de sedação ou anestesia geral. Este trabalho teve como objetivo comparar os efeitos sedativos do midazolam (5 mg. $\left.\mathrm{kg}^{-1}\right)$ associado ou não ao butorfanol (1 mg. $\left.\mathrm{kg}^{-1}\right)$ pelas vias de administração intranasal ou intramuscular. Sete periquitos australianos foram submetidos a quatro tratamentos em delineamento do tipo crossover com 15 dias de intervalo. Foram avaliados os períodos de latência, tempos de decúbito dorsal, tempos de sedação, tempos de recuperação, grau de sedação e qualidade de recuperação. Os resultados paramétricos foram avaliados por análise de variância de uma via seguida por teste de StudentNewman-Keuls e os dados não paramétricos foram submetidos ao teste Kruskal-Wallis ambos com $5 \%$ de significância. A técnica intranasal demonstrou melhores graus de sedação; no entanto, concluiu-se que ambos os protocolos e as vias de administração avaliados são seguras e viáveis para sedação em periquito australiano.
\end{abstract}

Palavras chaves: sedação, opioíde, benzodiazepínico, psitacídeo.

\begin{abstract}
Often veterinarians have attended various species of birds to perform clinical procedures, which require sedation or general anesthesia. The aim of this study was compare the intranasal or intramuscular sedative effects of midazolam $\left(5 \mathrm{mg}^{\mathrm{kg}} \mathrm{kg}^{-1}\right)$ with or without butorphanol (1 mg. kg-1). Seven budgerigards (Melopsitacus undulates) were submitted in a crossover design to four treatments. The procedures were performed with 15 days washout. Were evaluated the on set time, dorsal recumbency time duration, total sedation period, total recovery time, sedation degree and recovery quality. The parametric results were analised by one way ANOVA following Student-Newman-Keuls test and non-parametric datas were submitted to Kruskal-Wallis test, both with $5 \%$ significance. The intranasal technical demonstrates best degrees of sedation, however, this study concluded that both protocols and the administration routes are safe and viable for sedation in budgerigards.
\end{abstract}

Keywords: sedation, opioid, benzodiazepine, psittacines.

\section{Introdução}

Frequentemente os médicos-veterinários têm-se deparado com o desafio de atender variadas espécies de aves em suas rotinas, necessitando realizar diversos tipos de procedimentos, os quais muitas vezes requerem sedação ou anestesia geral, incluindo exame físico, radiográfico, colheita de material para exames complementares e procedimentos clínicos e cirúrgicos (Curro, 1998).

A maioria das espécies de aves são delicadas e reagem negativamente à contenção física por tempo prolongando, principalmente aquelas agitadas, doentes, debilitadas ou em diestresse respiratório (Lennox, 2011). Nesses pacientes, apenas a contenção física ou anestesia geral podem trazer riscos significantes, sendo a sedação uma alternativa segura para a realização de procedimentos e testes de diagnósticos (Lennox, 2011).

Para a anestesia e sedação de aves, é indicada a anestesia inalatória por ser considerada mais segura que a anestesia injetável, porém, faz-se necessário o uso de aparelhos com vaporizadores calibrados, os quais podem não estar disponíveis sempre que necessário (Vesal e Zare, 2006). Além disso, devido ao menor índice anestésico dos halogenados, quando utilizados em aves, a ocorrência de depressão respiratória e apneia pode ser comum (Ludders et al. 1990), sendo, portanto, necessária a monitoração constante e cuidadosa desses pacientes (Ludders, 2015).

Os anestésicos injetáveis podem ser administrados por via intramuscular, intraóssea, intravenosa ou através de cânula em saco aéreo e apresentam como vantagens o curto período de

*Recebido em 17 de março de 2016 e aceito em 29 de julho de 2017.

**Universidade Federal do Paraná - Setor Palotina, Palotina, Paraná, Brasil.

Autor para Correspondência: rafaelmessiasluiz@gmail.com 
latência, baixo custo, além de dispensarem o uso de aparelhos de anestesia inalatória (Kamiloglu et al. 2008). A administração de fármacos pela via intravenosa torna-se complexa e inviável em pacientes com massa corporal menor que 100 gramas, bem como administrações na musculatura da coxa podem gerar danos aos músculos e nervo ciático e ainda, o fármaco estará sujeito a sofrer efeito de primeira passagem pelo sistema portarenal (Ludders e Matthews, 1996).

A administração pelas vias intravenosa e intramuscular pode ser de difícil aplicação ou causar lesões nas aves e ainda acarretar em dor e estresse, principalmente se o fármaco possuir características irritantes (Vesal e Zare, 2006). Com o objetivo de eliminar os efeitos adversos da via intramuscular, Vesal e Eskandari (2006) e Bigham e Moghaddam (2009) recomendam o uso da via intranasal para a anestesia e sedação em aves, porém, estudos ainda são necessários para se estabelecer doses seguras e eficazes para utilização desta via nas diferentes espécies de aves.

A utilização de midazolam e outros fármacos por via intranasal foi descrita por Wilton et al. (1988) sendo segura e efetiva na redução do estresse e ansiedade em crianças. Em medicina veterinária, há relatos do uso da via intranasal em periquitos australianos (Trevisan et al. 2016), canários (Vesal e Zare, 2006), periquitos-de-colar (Vesal e Eskandari, 2006), mandarins (Bigham e Moghaddam, 2009), pombas-rola (Beier et al. 2013), em duas espécies de papagaios (Bitencourt et al. 2013) e pombas (Hornak et al. 2015).

Vários fármacos, isolados ou em associações, são utilizados para anestesia e sedação de aves. O midazolam produziu adequado relaxamento muscular e efeitos sedativos (Lierz e Korbel, 2012) com mínimos efeitos cardiopulmonares (Ludders e Matthews, 1996). Frequentemente, o midazolam é associado a anestésicos dissociativos e a opioides para acentuar os efeitos de relaxamento muscular e de sedação (Machin e Caulkett, 1998). Segundo Vesal e Zare (2006), a dose de midazolam intranasal em canários varia de $12,5 \mathrm{mg} \cdot \mathrm{kg}^{-1}$ a $15,6 \mathrm{mg} \cdot \mathrm{kg}^{-1}$, ao passo que Bigham e Moghaddam (2009) utilizaram 13 mg. $\mathrm{kg}^{-1}$ para obter sedação profunda em mandarins e Trevisan et al. (2016), utilizando $5 \mathrm{mg} \cdot \mathrm{kg}^{-1}$ de midazolam associado à cetamina, obtiveram sedação eficaz para procedimentos de curta duração.

O butorfanol é um opioide de ação mista, agonista-antagonista, de baixa atividade nos receptores $m u(\mu)$ e delta $(\delta)$ e forte interação agonista nos receptores kappa (K), características estas que levam à mínima depressão respiratória (Hawkins e Paul-Murphy, 2011). Em estudo com pombos-domésticos, Mansour et al. (1988) constataram a predominância de receptores kappa em relação aos receptores delta e $m u$, sugerindo que se os dados fossem extrapolados para outras espécies, o butorfanol seria o opioide de escolha para protocolos de anestesia e sedação em aves.

Além de causar pouca depressão respiratória, o butorfanol (1 mg. $\mathrm{kg}^{-1}$ ) quando administrado pela via intramuscular, resulta em menor requerimento de anestésicos inalatórios para manutenção anestésica em cacatuas (Curro et al. 1994) e galinhas-de-angola (Escobar et al. 2012).

Devido à grande variedade de espécies e suas diferentes características fisiológicas, estudos são necessários para determinar a segurança e eficácia da sedação intranasal para o uso clínico e cirúrgico em aves. O presente estudo teve como objetivo comparar os efeitos sedativos e a eficácia clínica do midazolam, associado ou não ao butorfanol, administrado pelas vias intramuscular ou intranasal em periquito australiano (Melopsittacus undulatus).

\section{Materiais e métodos}

Foram utilizados sete periquitos australianos (Melopsitacus undulatus) adultos jovens, hígidos, de ambos os sexos, alocados em viveiro coletivo com água e ração comercial ad libitum e submetidos a período de adaptação ambiental, alimentar e de manejo durante quatro semanas. No período de adaptação, as aves foram submetidas a exame clínico semanal, no qual era avaliado o escore corporal, a atitude e movimentação, inspeção de cloaca e narinas e ausência de alterações respiratórias.

Após o período de adaptação, as aves foram submetidas a quatro tratamentos em delineamento do tipo crossover, com intervalo de 15 dias entre os tratamentos. Foram avaliados dois protocolos de sedação: Midazolam (M) (midazolam 5,0 mg. $\mathrm{kg}^{-1}$ Midazolam $^{\circledR}$, Hipolabor Farmacêutica Ltda, Sabará, MG, Brasil) e midazolam associado ao butorfanol (MB) (midazolam 5 mg. $\mathrm{kg}^{-1}$ e butorfanol 1,0 mg. $\mathrm{kg}^{-1}$ - Turbogesic ${ }^{\circledR}$, Laboratório Fort Dodge, Campinas, SP, Brasil), os dois protocolos foram administrados pelas vias intranasal $(\mathrm{N})$ ou intramuscular $(\mathrm{M})$. Para o estudo, o butorfanol foi previamente diluído em água bidestilada estéril, para atingir a concentração de $2,5 \mathrm{mg} \cdot \mathrm{mL}^{-1}$.

Para a administração dos anestésicos, as aves foram mantidas em decúbito dorsal por meio de contenção física do tronco e membros pélvicos junto das asas, permitindo livre movimentação da musculatura intercostal e peitoral, a cabeça contida com a mesma mão da contenção corporal. Foram utilizadas seringas de 30 UI, BD Ultra-Fine II ${ }^{\mathrm{TM}}$, agulhadas. Nos grupos MM (midazolam administrado por via intramuscular) e MBM (associação de midazolam e butorfanol administrados por via intramuscular) a administração foi realizada nos músculos peitorais, enquanto nos grupos MN (midazolam via intranasal) e MBN (associação de midazolam e butorfanol administrados por via intranasal) os fármacos foram instilados lentamente e diretamente nas narinas, sendo que no grupo MBN cada fármaco foi administrado em uma narina. Não foi realizado jejum prévio em nenhuma ave.

Posterior à administração dos fármacos, as aves foram posicionadas sobre colchão térmico e foram avaliados pelo mesmo observador os períodos de latência (tempo decorrido entre a administração dos fármacos até a observação dos efeitos clínicos, caracterizado pela permanência em decúbito dorsal e redução dos reflexos protetores), tempos de decúbito dorsal (tempo decorrido entre o final do período de latência e a primeira tentativa de permanecer em estação), tempos de sedação (tempo decorrido entre o final do período de latência e a recuperação da sedação) e tempos de recuperação (períodos compreendidos do final do tempo de decúbito dorsal até que a ave consiga permanecer em estação e retomar suas atividades).

O grau de sedação foi avaliado após cinco minutos da administração dos fármacos através de uma escala em 4 graus (S0-S3), baseada nos estudos de Valverde et al. (1993) e Kamiloglu et al. (2008) onde: S0 é considerada a melhor sedação, ave com os olhos fechados, em decúbito, relaxado e sem movimentação; S1: ave com olhos abertos, em decúbito, relaxada e resposta leve a estímulos (manipulação e sons); S2: ave com olhos abertos, piscando, em decúbito, relaxada, com resposta a estímulos e movimentação leve; S3: ave acordada, ausência de decúbito e responsiva a estímulos. 
A qualidade da recuperação foi classificada conforme a escala de Kamiloglu et al. (2008) em três graus (R1-R3) onde R1 corresponde à ave quieta e tranquila, R2: ave com ataxia e tremores ocasionais, R3: ave com ataxia e tremores moderados.

Os resultados foram submetidos ao teste de normalidade de Kolmogorov-Smirnov e os dados paramétricos foram avaliados por análise de variância de uma via seguida por teste de StudentNewman-Keuls e os dados não paramétricos foram submetidos ao teste Kruskal-Wallis ambos com 5\% de significância.

O presente estudo foi aprovado pela Comissão de Ética no Uso de Animais da instituição de origem sob o protocolo 13/2013.

\section{Resultados}

Os períodos de latência (Tabela 1) avaliados não foram estatisticamente diferentes entre MBN e MM, mas foram diferentes de MN (124 \pm 44,4 segundos) e MBM (203 \pm 172 segundos) e também foram diferentes entre si.

Os tempos de decúbito dorsal foram significativamente diferentes entre os grupos MN, MBN e MM; no entanto, os grupos MM e MBM e os grupos MBM e MBN foram iguais entre eles, sendo o maior tempo de decúbito observado no grupo MN ( $9.9 \pm 7,19$ minutos).
Os tempos de sedação nos grupos MBN, MM e MBM foram iguais entre eles, porém diferiram do grupo $M N$, sendo os maiores tempos de sedação observados nos grupos submetidos à técnica de administração intramuscular.

Foi observada diferença nos tempos de recuperação (Tabela 1) entre $\mathrm{MN}(2,8 \pm 1,53$ segundos) e MBN (9,72 $\pm 3,91$ segundos), sendo que os grupos MM e MBM foram iguais entre eles, mas diferentes dos grupos MN e MBN. Os menores tempos de recuperação foram observados nos grupos em que foram submetidos à técnica de administração intranasal.

$\mathrm{Na}$ avaliação do grau de sedação (Tabela 2), apenas os grupos $\mathrm{MN}$ e MBN demonstraram sedação S0, enquanto no grupo MM $71,42 \%$ dos periquitos foram classificados em S2 e $28,56 \%$ em S1. No grupo MBM $28,56 \%$ das aves obtiveram sedação em S1 e $72,42 \%$ em S2. Nenhum dos indivíduos apresentou grau de sedação $S 3$.

Não houve diferença significativa entre os grupos na qualidade de recuperação (Tabela 2), sendo que em MN foi observado que $85,72 \%$ das aves obtiveram recuperação R1 e 14,28\% R2. Nos demais grupos, todos os indivíduos demostraram qualidade de recuperação $\mathrm{R} 1$.

Tabela 1: Médias e desvios padrão do período de latência, tempo de decúbito dorsal, tempo de sedação e tempo de recuperação de periquitos australianos (Melopsittacus undulatus) ( $\mathrm{n}=7$ ) submetidos a sedação com midazolam (M) ou midazolam e butorfanol (MB) pelas vias intranasal (N) ou intramuscular (M)

\begin{tabular}{|c|c|c|c|c|}
\hline & \multicolumn{2}{|c|}{ INTRANASAL } & \multicolumn{2}{|c|}{ INTRAMUSCULAR } \\
\hline & Midazolam (MN) & $\begin{array}{c}\text { Midazolam + Butorfanol } \\
(\mathrm{MBN})\end{array}$ & Midazolam (MM) & $\begin{array}{c}\text { Midazolam + Butorfanol } \\
(\text { MBM) }\end{array}$ \\
\hline Período de latência (segundos) & $124 \pm 44,4^{a}$ & $176 \pm 112^{b}$ & $242 \pm 125^{b}$ & $203 \pm 172^{c}$ \\
\hline Tempo de decúbito dorsal (minutos) & $9.9 \pm 7,19^{a}$ & $5,62 \pm 4,53^{c}$ & $6,6 \pm 14,25^{b}$ & $6,78 \pm 13,9^{b, c}$ \\
\hline Tempo de sedação (minutos) & $12,7 \pm 6,86^{a}$ & $15,3 \pm 2,79^{b}$ & $26,3 \pm 16,3^{b}$ & $24,0 \pm 13,6^{b}$ \\
\hline Tempo de recuperação (minutos) & $2,8 \pm 1,53^{a}$ & $9,72 \pm 3,91^{c}$ & $19,7 \pm 16,5^{b}$ & $17,2 \pm 6,75^{b}$ \\
\hline
\end{tabular}

Letras diferentes na mesma linha indicam diferença estatística entre os grupos.

Tabela 2: Porcentagens do grau de sedação e qualidade de recuperação de periquitos-australianos (Melopsittacus undulatus) $(n=7)$ submetidos a sedação com midazolam (M) ou midazolam e butorfanol (MB) utilizando as vias intranasal (N) ou intramuscular $(\mathrm{M})$

\begin{tabular}{|c|c|c|c|c|c|}
\hline & & \multicolumn{2}{|c|}{ INTRANASAL } & \multicolumn{2}{|c|}{ INTRAMUSCULAR } \\
\hline & & Midazolam (MN) & $\begin{array}{c}\text { Midazolan + Butorfanol } \\
(\text { MBN) }\end{array}$ & Midazolam (MM) & $\begin{array}{c}\text { Midazolam + Butorfanol } \\
\text { (MBM) }\end{array}$ \\
\hline \multirow{4}{*}{ GRAU DE SEDAÇÃO } & So & $1(14,28 \%)$ & $1(14,28)$ & $0(0 \%)$ & $0(0 \%)$ \\
\hline & S1 & $1(14,28 \%)$ & $5(71,42 \%)$ & $2(28,56 \%)$ & $2(28,56 \%)$ \\
\hline & $\mathrm{S} 2$ & $5(71,42 \%)$ & $1(14,28 \%)$ & $5(71,42 \%)$ & $5(71,42 \%)$ \\
\hline & S3 & $0(0 \%)$ & $0(0 \%)$ & $0(0 \%)$ & $0(0 \%)$ \\
\hline \multirow{3}{*}{ QUALIDADE RECUPERAÇÃO } & $\mathrm{R} 1$ & $6(85,72 \%)$ & $7(100 \%)$ & $7(100 \%)$ & $7(100 \%)$ \\
\hline & $\mathrm{R} 2$ & $1(14,28 \%)$ & $0(0 \%)$ & $0(0 \%)$ & $0(0 \%)$ \\
\hline & R3 & $0(0 \%)$ & $0(0 \%)$ & $0(0 \%)$ & $0(0 \%)$ \\
\hline
\end{tabular}

S0: ave com os olhos fechados, em decúbito, relaxado e sem movimentação; S1: ave com olhos abertos, em decúbito, relaxada e resposta leve a estímulos; S2: ave com olhos abertos, piscando, em decúbito, relaxada, com resposta a estímulos e movimentação leve; S3: ave acordada, ausência de decúbito e responsiva a estímulos. R1 corresponde à ave quieta e tranquila, R2: ave com ataxia e tremores ocasionais, R3: ave com ataxia e tremores moderados. 


\section{Discussão}

O estudo revelou que a via intranasal é eficaz para a administração de midazolam, associado ou não ao butorfanol, sendo observado curto tempo de latência, sedação de grau moderado e recuperação rápida. Os resultados encontrados corroboram com os trabalhos de Lierz e Korbel (2012), Moghadam et al. (2009) e Trevisan et al. (2016), que também avaliaram protocolos de sedação administrados pela via intranasal.

Os resultados obtidos em relação ao período de latência podem estar relacionados com a via de administração utilizada. Bitencourt et al. (2013) obtiveram resultados semelhantes quando compararam as técnicas de administração intranasal e intramuscular em papagaios, sendo que os tempos obtidos com o uso da técnica intranasal foram menores que os tempos obtidos com a técnica intramuscular, sugerindo uma rápida ação e absorção da via intranasal. Trevisan et al. (2016) também obtiveram maior período de latência para a administração pela via intramuscular quando comparado a via intranasal ao avaliar os efeitos anestésicos da administração de midazolam associado à cetamina, reforçando a teoria de que a via intramuscular requer maior tempo para absorção do anestésico.

Vesal e Zare (2006) obtiveram resultados semelhantes em relação ao período de latência (1,9 \pm 1,0 minutos) quando administraram midazolam (12,5 a $\left.15,6 \mathrm{mg} \cdot \mathrm{kg}^{-1}\right)$ pela via intranasal em canários (Serinus canarius). No entanto, o tempo de decúbito dorsal $(17,1 \pm 5,0$ minutos) e o tempo de sedação (36,9 \pm 5,3 minutos) foram maiores. Bigham (2013) também observou tempos de decúbito dorsal e sedação maiores, utilizando $13,2 \pm 1,3 \mathrm{mg} . \mathrm{kg}^{-1}$ de midazolam pela via intranasal em periquitos. Quando comparados aos trabalhos citados, os menores tempos de decúbito dorsal e sedação obtidos neste trabalho podem estar relacionados com a diferença entre as espécies e a dose de midazolam utilizada. Os menores tempos de decúbito dorsal e sedação obtidos na administração intranasal em relação a intramuscular podem estar relacionados com a rápida biotransformação resultante da administração dos fármacos pela via intranasal que acarreta em efeitos clínicos de curta duração em aves (Bigham e Moghaddam, 2009).

Quanto ao tempo de recuperação e às vias de administração utilizadas, Beier et al. (2013) utilizando cetamina S+e midazolam em pomba-rola, Bitencourt et al. (2013) empregando cetamina e midazolam em papagaios e Trevisan et al (2016) avaliando a associação de cetamina e midazolamem periquitos, também relataram menores tempos de recuperação das aves com o emprego da técnica intranasal em comparação à intramuscular, estes resultados estão relacionados com a rápida biotransformação da via intranasal (Bigham e Moghaddam, 2009).
Embora tenham utilizado um protocolo de anestesia dissociativa, Bitencourt et al. (2013) obtiveram melhores graus de sedação com o emprego da técnica intranasal comparado a técnica intramuscular, assim como em nosso trabalho, sugerindo que esta é uma eficiente via de administração para sedação em aves. O grau de sedação leve (S2) observado na maioria dos indivíduos tratados por via intramuscular pode estar relacionado com a absorção dos fármacos por esta via, uma vez que na administração de fármacos injetáveis pode haver variação significativa intraespecífica em termos de dose e resposta (Ludders, 2015).

A diferença no grau de sedação entre os grupos $\mathrm{MN}$ e MBN, foi relacionada com o uso em associação do midazolam e butorfanol, que atuam de forma sinérgica, promovendo a potencialização dos seus efeitos sedativos (Gunkel e Lafortune, 2005). Hornak et. al. (2015) também observaram discrepância de resultados quando compararam o uso isolado de midazolam associado ou não à dexmedetomidina; neste caso, os indivíduos que foram tratados apenas com midazolam obtiveram menor grau de sedação com resposta a estímulos.

Assim como no presente trabalho, Vesal e Eskandari (2006) não observaram complicações na recuperação de periquitosde-colar após administração de $7,3 \mathrm{mg} \cdot \mathrm{kg}^{-1}$ de midazolam pela via intranasal, estes autores relatam que as aves sedadas com midazolam intranasal têm recuperação ativa e interesse em se alimentar, características também observadas em nosso trabalho.

Observou-se durante a administração intranasal que $71,42 \%$ dos periquitos em MBN e 42,85\% em MN espirraram durante a recuperação, fato que pode estar relacionado com os mecanismos de defesa do sistema respiratório (Grassin-Delyle et al, 2012). Em MN, um dos indivíduos apresentou cianose leve e dois apresentaram tremores leves, dentre os quais um deles apresentou respiração abdominal. No mesmo grupo, um indivíduo manifestou excitação durante a recuperação. Em MM dois indivíduos apresentaram excitação e tremores leves, podendo estes sinais serem decorrentes de excitação paradoxal promovida pelo midazolam (McKenzie e Rosenberg, 2010).

Concluindo, o uso do midazolam associado ou não ao butorfanol e administrado pelas vias intranasal ou intramuscular nas doses propostas mostrou-se eficaz e seguro para a sedação de periquitos australianos e pode ser utilizado para a contenção química de periquitos. A técnica intranasal apresentou melhores graus de sedação e a associação do midazolam ao butorfanol administrados pela via intranasal pode ser útil para sedação de pacientes agitados e estressados para realização de procedimentos indolores de curta duração. 


\section{Referências}

AJADI, R.A; KASALI, O.B; ADELEYE, A.I; OYEWUSI, J.A, AKINTUNDE, O.G. Effects of midazolam on ketamine-xylazine anesthesia in guinea fowl (Numida meleagris galeata). Journal of Avian Medicine and Surgery, v. 23, n. 3, p. 199 - .

BEIER, S.L; ROSA, A.C; OLESKOVICZ, N; MATTOSO, C.R.S; MORAES, A.N. Efeitos anestésicos da administração intranasal ou intramuscular de cetamina $S+$ e midazolan em pomba-rola (Streptotelia sp.). Pesquisa Veterinária Brasileira, v. 33, n. 4, p. 517-522, 2013.

BIGHAM, A.S. Comparison of intranasal administration of xylazine, diazepam, and midazolam in budgerigars (melopsittacus undulatus): clinical evaluation. Journal of Zoo and Wild Life Medicine, v. 44, n. 2, p. 241-244, 2013.

BIGHAM, A.S; MOGHADDAM, Z.A.K. Finch (Taeneopygia guttata) sedation with intranasal administration of diazepam, midazolam or xylazine. Journal of Veterinary Pharmacology and Therapeutics, v. 36, n.1, p. 1- 3, 2009.

BITENCOURT, E.H; PADILHA, V.A; LIMA, M.P.A; BEIER, S.L; MORAES, A.N; OLESKOVICZ, N. Efeitos sedativos da associação de cetamina e midazolam administrados pela via intranasal ou intramuscular em papagaio (Amazona aestiva e Amazona vinacea). Pesquisa Veterinaria Brasileira, v. 33, n. 9, p. 1125-1129, 2013.

CURRO, T.G. Anesthesia of pet birds. Seminars in Avian and Exotic Pet Medicine, v. 7, n.1, p. 10-21, 1998.

CURRO, T.G; BRUNSON, D.B; PAUL-MURPHY, J. Determination of the ED50 of isoflurane and evaluation of the isoflurane-sparing effects of butorphanol in cockatoos (Cacatua spp.). Veterinary Surgery, v. 23, p. 429 -433, 1994.

DAY, T.K; ROGE, C.K. Evaluation of sedation in quail induced by use of midazolam an reversed and reversed by use flumazenil. Journal of America Veterinary Medical Association. v. 209, n.5, p. 969-971, 1996.

ESCOBAR, A; VALADÃO, C.A.A; BROSNAN, R.J; DENICOL, A.C; FLÔRES, F.N; THIESEN, R; COELHO, C.M.M. Effects of butorphanol on the minimum anesthetic concentration for sevoflurane in guineafowl (Numida meleagris). American Journal of Veterinary Researches, v. 73, n. 2, p. 183-188, 2012.

FORBES, N.A. Avian Anesthesia. Veterinary Quarterly, v. 20, p. 65-66, 1998.

GRASSIN-DELYLE, S; BUENESTADO, A; NALINE, E; FAISY, C; BLOUQUIT-LAYE, S; COUDERC, L. Intranasal drug delivery: an efficient and non-invasive route for systemic administration: focus on opioids. Pharmacology \& Therapeutics, v. 134, n. 3, p. 66-79, 2012.

GUNKEL, C; LAFORTUNE, M. Current techniques in avian anesthesia. Seminars in Avian an Exotic Pet, v. 14, n. 4, p. 263276, 2005.

HAWKINS, M.G; PAUL-MURPHY, J. Avian Analgesia. Veterinary Clinics Exotic Animals, v. 14, p. 61-80, 2011.

HORNAK, S; LIPTAK, T; LEDECKY, V; HROMADA, R; BILEK, $\mathrm{J}$; DAVID MAZENSKY, D; PETROVIC, V. A preliminary trial of the sedation induced by intranasal administration of midazolam alone or in combination with dexmedetomidine and reversal by atipamezole for a short-term immobilization in pigeons.

Association of Veterinary Anaesthetists and the American College of Veterinary Anesthesia and Analgesia, v. 42, p. 192-196, 2015.
KAMILOGLU, A; ATALAN, G; KAMILOGLU, NN. Comparison of intraosseous and intramuscular drug administration for induction of anaesthesia in domestic pigeons. Research in Veterinary Science, v. 85, n. 1, p. 171-175, 2008.

LENNOX, A.M. Sedation as an alternative to general anesthesia in pet birds. In: PROCEEDINGS OF THE EUROPEAN ASSOCIATION OF AVIAN VETERINARIANS. Madrid, 2011.

LICHTENBERHER, M; KO, J. Anesthesia and analgesia for small mammals and birds. Veterinary Clinics Exotc Animal Pratice, v. 10, p. 293-315, 2007.

LIERZ, M; KORBEL, R. Topics in Medicine and Surgery Anesthesia and Analgesia in Birds. Journal of Exotic Pet Medicine, v. 21, p. 21-44, 2012.

LUDDERS, J.W. Comparative Anesthesia and Analgesia of Birds. In: GRIMM, K.A; LAMONT, L.A; TRANQUILLI, W.J; GREENE, S.A; ROBERTSON, S;A (eds.) Veterinary Anesthesia and Analgesia. 5ed. United States of America: Wiley Blackwell, 2015, p. $800-816$.

LUDDERS, J.W; MATT .645-669, 1996.

LUDDERS, J.W; MITCHELL, G.S; RODE, J.B. Minimal anesthetic concentration and cardiopulmonary dose response of isoflurane in ducks. Veterinary Surgery, v. 19, n. 4, p. 304-307, 1990.

MACHIN, K.L; CAULKETT, N.A. Effects of injectable anesthetics in mallard ducks (Anas platyrhynchos): A descriptive study. Journal of Avian Medicine and Surgery, v. 12, n. 4, p. 244-262, 1998.

MANS, C. Sedation of pet birds. Journal of Exotic Pet Medicine, v. 23, p. 153-157, 2014.

MANSOUR, A; KHACHATURIAN, H; LEWIS, M.E; AKIL, H; WATSON, S.J. Anatomy of CNS opioid receptors. Trends in Neurosciences, v. 11, n. 7, p. 308-314, 1988.

MCKENZIE W.S; ROSENBERG M. Paradoxical reaction following administration of a benzodiazepine. International Journal of Oral \& Maxillofacial Surgery. n.68, p. 3034-3036, 2010.

MOGHADAM, A.Z; SADEGH, A.B; SHARIFI, S; HABIBIAN, S. Comparison of intranasal administration of diazepam, midazolam and xylazine in Pigeons: Clinical evaluation. Iranian Journal of Veterinary Science and Technology v. 1, n. 1, p. 19-26, 2009.

TREVISAN, G.A; SILVA, E.L; CARVALHO, A.L; LUIZ, R.M; efeitos anestésicos da administração intranasal ou intramuscular da associação de midazolam e cetamina racêmica ou $\mathrm{s}+$ em periquito australiano (melopsittacus undulatus). Ciência Animal Brasileira, v. 17, n. 1, p. 126-132, 2016.

VALVERDE, A; BIENZLE, D; SMITH, D.A; DYSON, D.H; VALLIANT, A.E; Intraosseous cannulation and drug administration for induction of anesthesia in chickens. Veterinary Surgery, v. 22, n. 3, p. 240-244, 1993.

VESAL, N; ESKANDARI, M.H. Sedative effects of midazolam and xylazine with or without ketamine and detomidine alone following intranasal administration in Ring-necked Parakeets. Journal of the American Veterinary Medical Association, v. 228, n. 3, p. 383-388, 2006.

VESAL, N; ZARE, P. Clinical evaluation of intranasal benzodiazepines, $\propto_{2}$-agonista and their antagonists in canaries. Veterinarian Anesthesia and Analgesia, v. 33, p. 143- 148, 2006.

WILTON, N.C.T; LEIGHT, J; ROSEN, D.R; PANDIT, A. Preanesthetic sedation of preschool children using intranasal midazolam. The Journal of American Socity of Anesthesiologists, v. 69, p. $972-974,1988$. 